\title{
Rare earth elements (REE) in biology and medicine
}

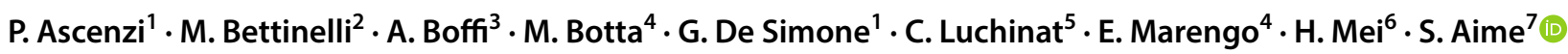

Received: 12 May 2020 / Accepted: 3 July 2020 / Published online: 16 July 2020

(c) The Author(s) 2020

\begin{abstract}
This survey reports on topics that were presented at the workshop on "Challenges with Rare Earth Elements. The Periodic Table at work for new Science \& Technology" hold at the Academia dei Lincei in November 2019. The herein reported materials refer to presentations dealing with studies and applications of rare earth elements (REE) in several areas of Biology and Medicine. All together they show the tremendous impact REE have in relevant fields of living systems and highlight, on one hand, the still existing knowledge gap for an in-depth understanding of their function in natural systems as well as the very important role they already have in providing innovative scientific and technological solutions in a number of biomedical areas and in fields related to the assessment of the origin of food and on their manufacturing processes. On the basis of the to-date achievements one expects that new initiatives will bring, in a not too far future, to a dramatic increase of our understanding of the REE involvement in living organisms as well as a ramp-up in the exploitation of the peculiar properties of REE for the design of novel applications in diagnostic procedures and in the set-up of powerful medical devices. This scenario calls the governmental authorities for new responsibilities to guarantee a continuous availability of REE to industry and research labs together with providing support to activities devoted to their recovery/recycling.
\end{abstract}

Keywords REE $\cdot$ Lanthanides $\cdot$ Biomedical applications $\cdot$ Lanthanide-based assays

This peer-reviewed paper belongs to the Topical Collection "The Periodic System". It is based on the presentations given at the International Conference organized by Accademia Nazionale dei Lincei and Fondazione "Guido Donegani": "Challenges with Rare Earth Elements. The Periodic Table at work for new Science \& Technology" held at the Accademia Nazionale dei Lincei in Rome, 14-15 November 2019 (coordinated by Silvio Aime and Roberta Sessoli).

S. Aime

silvio.aime@unito.it

Dipartimento di Scienze, Università Roma Tre, Rome, Italy

2 Dipartimento di Biotecnologie, Università di Verona, Verona, Italy

3 Dipartimento di Biochimica, Università "Sapienza" Roma, Rome, Italy

4 Dipartimento di Scienze e Tecnologie, Università del Piemonte Orientale, Alessandria, Italy

5 Dipartimento di Chimica, Università di Firenze, Florence, Italy

6 Deutsches Reuma-Forschungszentrum, a Leibniz Institute, Berlin, Germany

7 Dipartimento di Biotecnologie Molecolari e Scienze per la Salute, Università di Torino, Turin, Italy

\section{Introduction}

The group of REE consists of 17 representatives including Lanthanum, the Lanthanoids, Scandium and Yttrium. Another common name for the elements from $\mathrm{La}$ to $\mathrm{Lu}$ $(Z=57-71)$ is Lanthanides ( $\mathrm{Ln})$. Most of them are not rare at all; for instance, Cerium is the 25th most abundant element (more abundant than $\mathrm{Sn}$ and $\mathrm{Pb}$, just after $\mathrm{Zn}$ ). "Rare" relates to the considerable difficulties in separating one REE from another because of their close similarity in chemical and physical properties. "Earth", an old chemical name for oxides, was applied because they were first identified as rare earth oxides. REEs, often indicated as the "treasury" of new materials, play a key role in generating innovation in manufacturing industry, in a number of traditional and high technology products. Their peculiar electronic structure with $4 \mathrm{f}$ orbitals buried inside the atoms and shielded by $5 \mathrm{~s}$ and $5 \mathrm{p}$ electrons endow them with unique catalytic, magnetic and electronic properties. In the following, the charge or oxidation state of the REE will be given, as it is customary, either with a subscript or with a roman number in parentheses. This will not imply a specification of the type of bond linking the REE with the surroundings. 


\section{Lanthanides in living systems}

Over the last decade, rare earth elements (REEs) have been reported as essential elements for life. Although we are still far from achieving a detailed picture of the complex interactions/interferences brought by REE in living systems there is a growing attention on the potential role they may have as antagonists of important elements such as Calcium and Zinc and as catalytic centers of metalloenzymes. The supply of REEs to favor the growth of plants as well as in feeding animals is a patrimony of the traditional Chinese agriculture (e.g., $\mathrm{La}^{3+}$ and $\mathrm{Ce}^{3+}$ are used for stimulating crop rooting, germinating and increase crop chlorophyll content). The "normosis" effect for which positive effects are obtained only at given concentrations of the administered doses is the result of a complex interplay among metal complexes equilibria affecting the cellular metabolism and the overall level of the organ and organisms (Fashui 2002; Hibi et al. 2011; Nakagawa et al. 2012; Wang et al. 2012; Zhao et al. 2013; Chen et al. 2015; Chistoserdova 2016; Ramírez-Olvera et al. 2018).

Lanthanides $\left(\mathrm{Ln}^{3+}\right)$ have been reported to act as antagonists of $\mathrm{Ca}^{2+}$ blocking both voltage operated and receptor calcium channels (Agarwal and Kalra 1983; Fricker 2006; Ene et al. 2015). Although to date the mechanism by which the antagonist effects of $\mathrm{Ln}^{3+}$ are not yet clear, their actions depend on the similarity between the $\mathrm{Ln}^{3+}$ radii and that of $\mathrm{Ca}^{2+}$; of note, the inhibitory effect of $\mathrm{Ln}^{3+}$ increases with their ionic radius. Interestingly, $\mathrm{La}^{3+}, \mathrm{Nd}^{3+}$, $\mathrm{Sm}^{3+}, \mathrm{Eu}^{3+}, \mathrm{Tb}^{3+}$ and $\mathrm{Dy}^{3+}$ inhibit the uptake of $\mathrm{Ca}^{2+}$ in Mycobacterium phlei through a non-competitive inhibition mechanism which does not interfere with the respiration process and membrane-bound ATPase activity (Agarwal and Kalra 1983). Moreover, $\mathrm{Ln}^{3+}$ cations block the entry of $\mathrm{Ca}^{2+}$ into the cytosol of Saccharomyces cerevisiae cells only when they are present at a concentration high enough to permit a rapid binding to cell membrane; in addition, it has been speculated that plasma membrane $\mathrm{Ca}^{2+}$-channel Cch1/Mid1 contribute to $\mathrm{La}^{3+}$ entry into the yeast cells (Ene et al. 2015). Furthermore, in plant cells, it has been hypothesized that $\mathrm{Gd}^{3+}$ may reside in the mitochondrial intermembrane space blocking ion-channels of the mitochondrial inner membrane through a thiol chelation (Zhao et al. 2013).

Over the last decade, much attention has been paid to the role of $\mathrm{Ln}^{3+}$ in enzymology. Methylacidiphilum fumariolicum, found in the Solfatara volcanic crater (Napoli, Italy), is the only known bacterium unable to live in the absence of lanthanides. This bacterium grows at acidic $\mathrm{pH}$ 2-5 and high temperature $\left(50-60{ }^{\circ} \mathrm{C}\right)$ and uses methanol as a source of energy, which is converted to formaldehyde by $\mathrm{Ce}^{3+}$ - and $\mathrm{Ca}^{2+}$-dependent methanol dehydrogenases
(MDHs) (Nakagawa et al. 2012; Picone and Op den Camp 2019). MDHs, present in the obligate and facultative methylotrophs as well as in methanotrophs (Williams et al. 2005; Pol et al. 2014), are hetero-tetrameric enzymes that are characterized by two large $\alpha$ subunits $(\mathrm{MxaF})$ and two small $\beta$ subunits (MxaI). Each MxaF subunit binds pyrroquinoline quinone (PQQ) as the cofactor, which participates to the coordination of the metal ion, whereas the role of the MxaI subunits is unknown (Williams et al. 2005; Pol et al. 2014).

Methylacidiphilum fumariolicum expresses both a $\mathrm{Ca}^{2+}$ dependent MxaF-MDH and a $\mathrm{Ce}^{3+}$-dependent XoxF1-MDH (Nakagawa et al. 2012; Pol et al. 2014). The expression of the $\mathrm{Ca}^{2+}$ and the $\mathrm{Ce}^{3+}$-dependent subunit depends on the bioavailability of metal ions present in the environment (Fitriyanto et al. 2011; Hibi et al. 2011; Pol et al. 2014). The three-dimensional structure of the $\alpha$ subunits of $\mathrm{Ca}^{2+}$ and $\mathrm{Ce}^{3+}$-dependent MDH (MxaF and XoxF1, respectively) is characterized by an eight-bladed $\beta$-propeller; all blades (named W1-W8) are built from four antiparallel $\beta$-strands (named A-D) (Fig. 1) (Ghosh et al. 1995; Anthony 2004; Keltjens et al. 2014; Pol et al. 2014). $\mathrm{Ca}^{2+}$-dependent MxaFMDH from Methylobacterium extorquens (PDB ID: 1H4I) (Ghosh et al. 1995) and $\mathrm{Ce}^{3+}$-dependent XoxF-MDH from Methylacidiphilum fumariolicum (PDB ID: 4MAE) (Pol et al. 2014) have been taken as the prototypes of $\mathrm{Ca}^{2+}$ dependent and $\mathrm{Ce}^{3+}$-dependent MDHs, respectively.

In the $\mathrm{Ca}^{2+}$-dependent MxaF-MDH from Methylobacterium extorquens, the metal ion is coordinated by the OD1 oxygen of Asn261, the OE1 and OE2 oxygen atoms of Glu177, and the O5, N6, and O7 atoms of the PQQ cofactor (Ghosh et al. 1995; Nojiri et al. 2006), whereas in $\mathrm{Ce}^{3+}$ dependent XoxF-MDH from Methylacidiphilum fumariolicum, the metal ion is coordinated by the OD1 oxygen of Asn256, the OE1 and OE2 oxygen atoms of Glu172, the O5, N6, and $\mathrm{O} 7$ atoms of the PQQ cofactor, the OD1 oxygen of Asp299, and the OD1 and OD2 oxygen atoms of Asp301. To facilitate the accommodation of the larger $\mathrm{Ce}^{3+}$ ion in the PQQ hydrophobic pocket of XoxF1-MDH from Methylacidiphilum fumariolicum, the Pro264 and Ala176 of $\mathrm{Ca}^{2+}-$ dependent MxaF-MDH from Methylobacterium extorquens are replaced by Thr259 and Gly171, respectively (Fig. 1) (Pol et al. 2014; Keltjens et al. 2014).

The Asp301 residue present only in $\mathrm{Ce}^{3+}$-dependent XoxF1-MDHs plays a pivotal role in $\mathrm{Ce}^{3+}$ selective binding. Notably, in the $\mathrm{Ce}^{3+}$-dependent XoxF1-MDHs the lanthanide ion forms eight coordination bonds in contrast to the five coordination bonds which stabilize the $\mathrm{Ca}^{2+}$ ion in $\mathrm{Ca}^{2+}$ dependent MxaF-MDHs. The absence of Asp301 in the $\mathrm{Ca}^{+2}$ dependent MxaF-MDHs avoids the coordination saturation of the $\mathrm{Ca}^{2+}$ ion, which would reduce the substrate affinity, as also indicated by density functional theory calculations (Bogart et al. 2015). Moreover, the lower Lewis acidity of 
Fig. 1 Three-dimensional structures of the $\mathrm{Ca}^{2+}$-dependent-MxaF-MDH from Methylobacterium extorquens (PDB ID: 1H4I) (Ghosh et al. 1995) and of the $\mathrm{Ce}^{3+}$-dependent-XoxF1-MDH from Methylacidiphilum fumariolicum SolV (PDB ID: 4MAE) (Pol et al. 2014). For each structure, the metal ion coordination environment is shown. The eight blades are highlighted with different colors and named W1-W8. Each blade is built by four antiparallel $\beta$-strands. PQQ is in black; the $\mathrm{Ca}^{2+}$ and $\mathrm{Ce}^{3+}$ ions are in orange red and blue, respectively. Dashed lines indicate the coordination bonds of metal ions
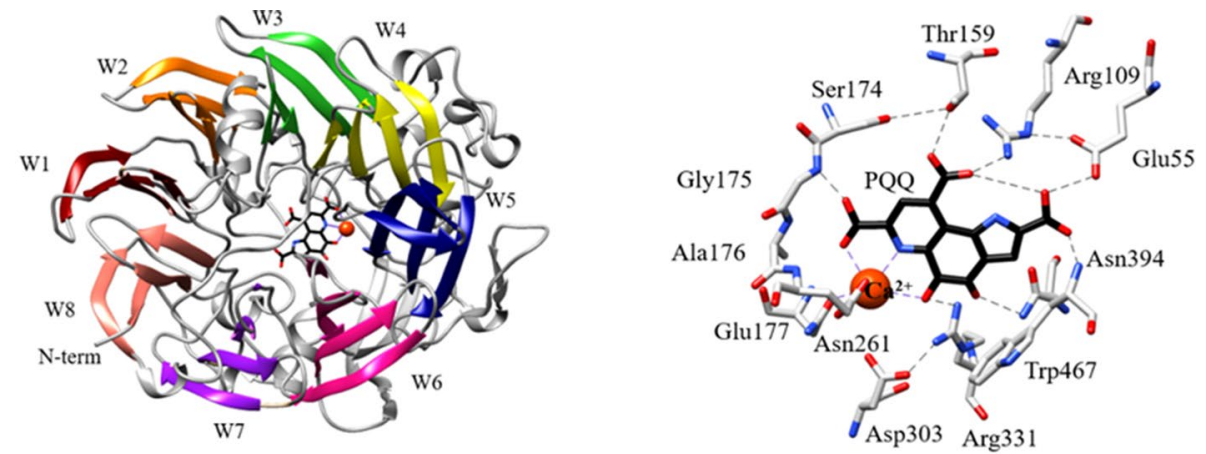

$\mathrm{Ca}^{2+}$-dependent MxaF-MDH from Methylobacterium extorquens
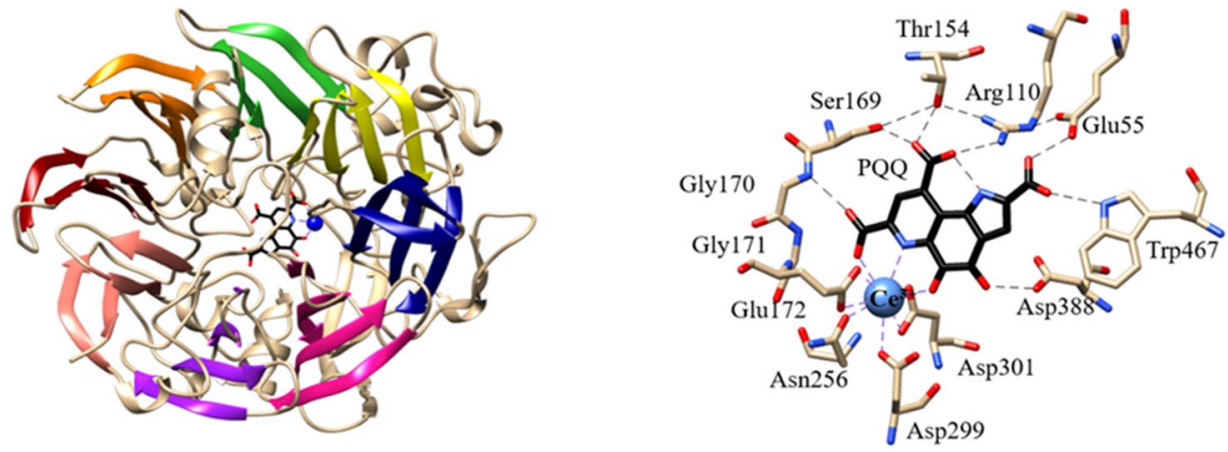

$\mathrm{Ce}^{3+}$-dependent XoxF-MDH from Methylacidiphilum fumariolicum
$\mathrm{Ca}^{2+}$ compared to $\mathrm{Ce}^{3+}$ influences the redox cycling of the PQQ cofactor (Bogart et al. 2015).

Studies so far, suggest that there is no evidence on the existence of lanthano-enzymes not PQQ dependent. In addition, the MDH family should be considered as typical of Archea and Bacteria (De Simone et al. 2019).

In conclusion, although recent biochemical studies attribute to lanthanides a key role in the biology of bacteria, many aspects of the biology of lanthanides have yet to be clarified. The identification of structural differences between the active sites of $\mathrm{Ca}^{2+}$-dependent-XoxF-MDH and $\mathrm{Ce}^{3+}$-dependent MxaF-MDH can be biotechnologically interesting to lead to new oxidation catalysts, finding application in fuel cells and fine chemical syntheses (Daumann, 2019; Cotruvo, 2019).

\section{Lanthanides in foodstuffs traceability and authentication}

Lanthanides are widely exploited in food authentication and traceability studies (Ballabio et al. 2018; Manfredi et al. 2018; Aceto et al. 2013; Marengo et al. 2005; Aceto 2016; Aceto et al. 2009, 2017, 2019; Oddone et al. 2009). The first ones are devoted to distinguish food differences related to the geographical provenance, the botanical or animal variety, the technological process of production or the coherence to the disciplinary of production. Traceability studies are instead devoted to identify the link between food and the soil from which the raw materials derive. Usually, traceability is guaranteed through a process of autocertification from the producer. An objective certification based on measured chemical-physical parameters should also be associated with this documentary certification; this characterization should rely on physical/chemical parameters supposed to remain unchanged along the production chain.

Authentication can be achieved through different chemical fingerprinting tools: elemental profiling, characterization of volatile molecules (electronic nose, volatile profile through SPME GC-MS), OMICS approaches (proteomics, genomics, metabolomics, volatilome etc.), spectroscopic techniques. This is possible since authentication is based on the characterization of the "authentic" food to distinguish it from other similar products. Traceability instead, which is based on the link between the food and the corresponding soil, can be guarantee by the use of the elemental profile, in particular the rare earths profile.

The plants absorb these elements from soil and water and distribute them to all their constituents (roots, leaves, fruits, etc.). It follows that the concentration profile present in a given soil is found also in the plants that grow on it thus making it an effective geographical fingerprint marker. The 
ability of not being fractionated from soil to plant and fruits makes REEs excellent candidates for traceability studies, where the link between foodstuffs and soils is verified along all the production chain.

Several applications have already been reported, spanning from wines to milk, olive oil, hazelnuts etc. In principle, the possibility to guarantee traceability along a production chain depends first on the length of the chain: in very short chains, as in the case of truffles, traceability is quite easy to demonstrate since the gap between soil and product is very short; in other cases, as for wine, milk etc. it must be verified case by case. An additional problem is due to the concentration levels of the REEs that must be determined, since the concentration decreases along the production chain; a typical example is given by wine, where the concentration decreases from $\mathrm{mg} / \mathrm{kg}$ in soil, to $\mu \mathrm{g} / \mathrm{kg}$ in grapes, $\mu \mathrm{g} / \mathrm{L}$ in musts and $\mathrm{ng} / \mathrm{L}$ in the final product.

Practically, the method relies on: (i) collection and mineralization of the specimens and (ii) quantitative determination of the REE (usually determined by ICP-MS) and (iii) set up of a multivariate statistical model which allows to assign the geographical origins. The last phase of model building usually exploits multivariate chemometric methods able to take into account the correlations existing between the variables, among them the REEs relative concentrations, is a very important one. The first step in data treatment is data normalization, particularly important in this context since REEs must be considered as profiles. To this purpose, they are usually considered as ratios calculated with reference to the most concentrated REE (cerium or lanthanum). An alternative approach is to consider all the possible ratios between one element with respect to all the others thus increasing the amount of information introduced in the models. Since the variables can show important scale effects, autoscaling is usually applied before multivariate analysis, consisting in the subtraction of the average value and normalization to unit variance. With this normalization all the variables gain the same importance. In all the cases, great attention must be paid to the predictive ability of the models as the aim is to obtain models able to correctly classify new samples and, in the meantime, able to include all the relevant information. To this purpose, internal and external validation procedures must be applied, and usually variable selection procedures are used to identify the most informative set of variables with the best predictive ability.

\subsection{Applications}

Several applications of REEs profile determination in traceability and authentication studies are present in literature, reporting different applications to short and medium production chains Herein we report some in-house examples on foodstuff production chains, investigated in our lab.

\subsubsection{Truffles}

Truffles represent a perfect case for the development of a traceability study aimed at identifying the highly valuable white truffles (as those ones from the Piedmont, Italy) from others. White and black truffles from different areas in Piedmont underwent incineration and the REE content was determined by CP-MS. The REE profile in truffles was compared to the ones from the corresponding soils obtaining very good results. The data were treated by PCA including truffles from Switzerland. Figure 2 reports the scores of the first two PCs calculated. The truffles from Switzerland appear well separated from the others at positive scores along both PC1 and PC2, proving the ability of the REE profile to separate the samples according to the provenance. Linear Discriminant Analysis was then applied to discriminate Piedmont from Swiss samples; the method was trained in forward search and the final model identified three markers as the most significant. Figure 3 reports the samples divided in the two classes according to the three ratios identified as relevant.

\subsubsection{Hazelnuts}

The production chain of hazelnut paste was investigated, taking into consideration three different cultivars, namely Mortarelle from Campania, Romane from Lazio and Piemonte IGP from Piedmont (this last one being the most valuable one). The production chain was investigated by considering raw and roasted hazelnuts, and hazelnut paste. The samples were mineralized by microwave assisted digestion adding nitric acid and hydrogen peroxide. The elemental analysis was accomplished by ICP-OES and ICP-MS for the determination of 48 isotopes. Data normalization was applied by calculating the ratio of each analyte with respect to all the

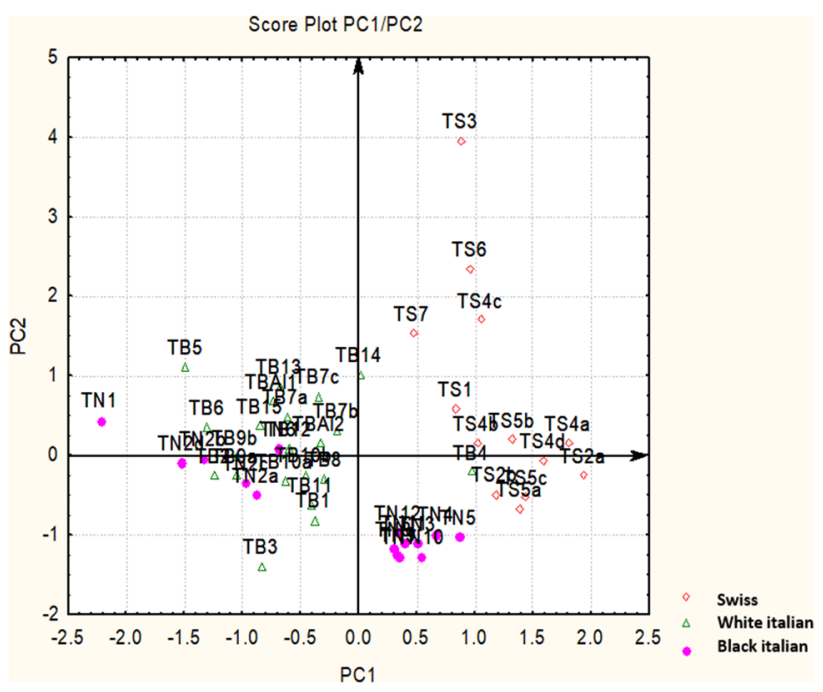

Fig. 2 Score plot of the first two PCs calculated on the truffle dataset 
Fig. 3 Truffle samples separated in Italian and Swiss by the three most discriminating variables identified by LDA

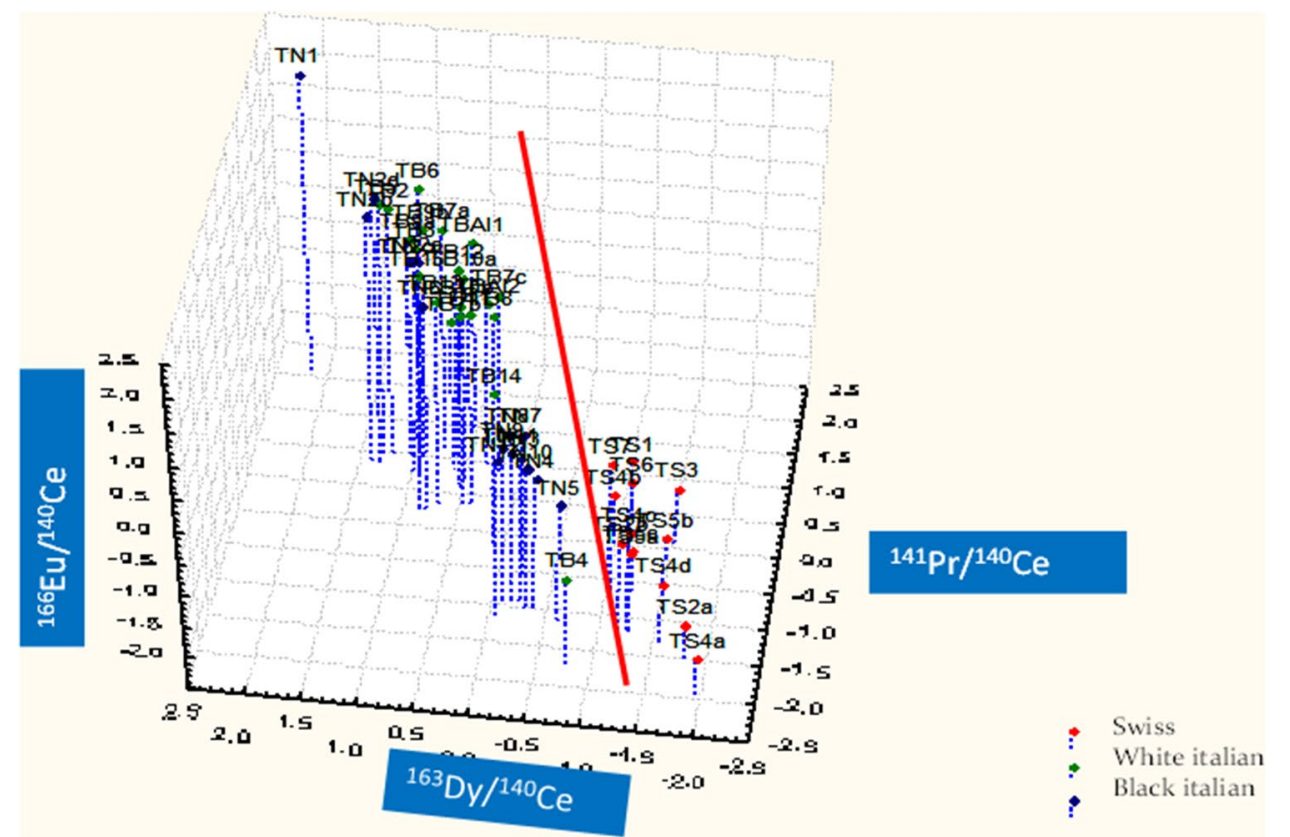

others; to eliminate the information about the type of product (raw, roasted, paste), the data were further centered for each type separately and autoscaling was applied on the overall data matrix. Figures 3 and 4 reports the score plot obtained by the first two PCs calculated (PC1 $30 \%$ of explained variance; PC2 about 20\%): the samples appear well separated along the first PC according to the cultivar: Piemonte IGP at negative values, Romane at positive ones and Mortarelle located at the origin of the axes. PLS-DA was applied on the same dataset with variable selection in backward elimination (smallest error in cross-validation- $20 \%$ of the objects excluded from the model at each cycle-1000 iterations); at each iteration the variables characterized by the smallest VIP score were eliminated. The final model contained 687 ratios and allowed the perfect classification of the samples in fitting and very good results in cross-validation (accuracy $>97 \%$, NER $\%>98 \%$ ).
Fig. 4 Score plot of the first two PCs calculated on hazelnut samples

\section{Scores plot hezelnuts}

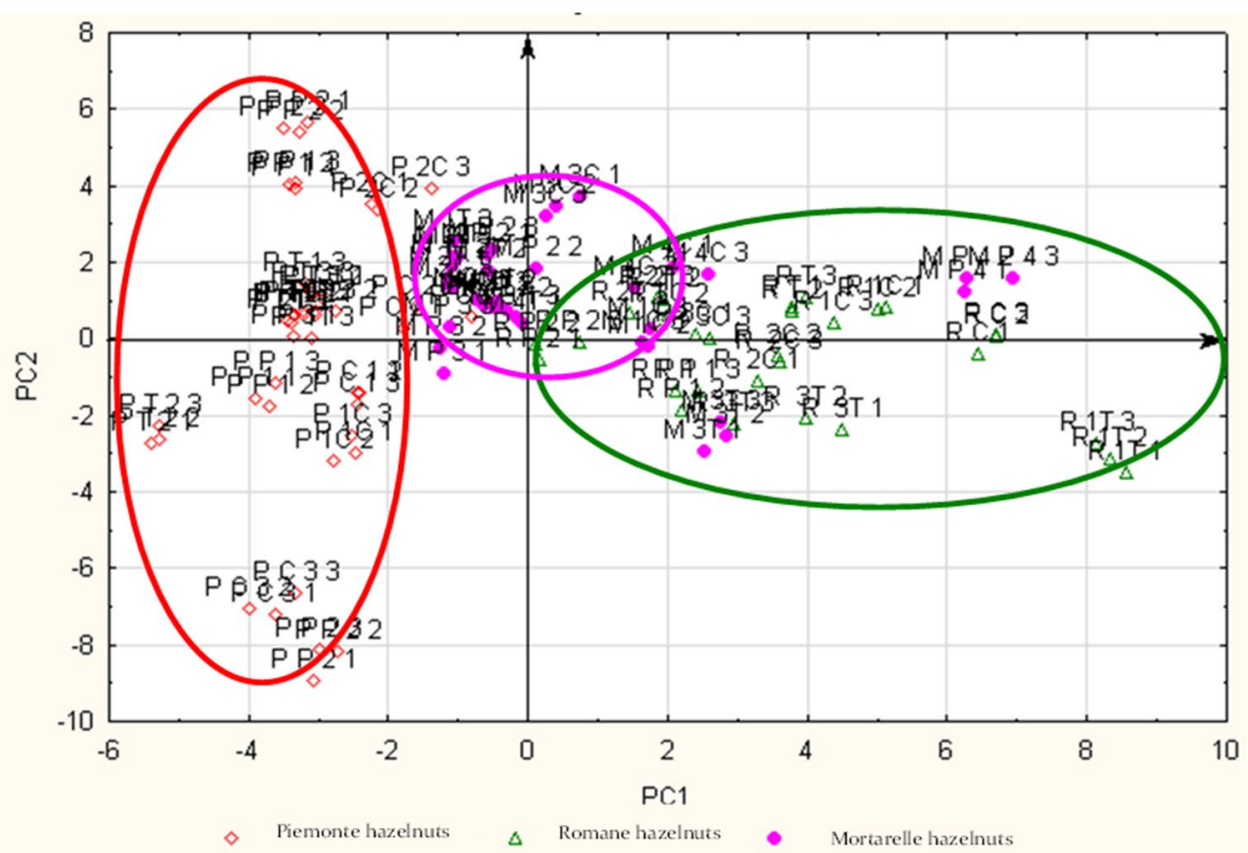




\subsubsection{Moscato wine}

Wine results from a quite long production chain involving several treatments that may interfere with the traceability information. To this purpose, the elemental profile of soils, grapes and musts from Moscato wine was compared (Aceto et al. 2013). All samples came from a pilot vineyard located in Piedmont. The REE profile from soils to grapes and musts proved to remain unaltered, while, as expected, the REE concentration decreased along the production chain. The last two steps involved filtration by diatomaceous earth and clarification through bentonites: the analysis of the results by PCA proved that these two steps altered the REE profile, releasing REEs in the musts from the diatomaceous earth and the bentonites used during the process. Therefore, in this case, the alteration of the REEs profile along the production chain hampers the possibility to guarantee the traceability. The study showed however that, while traceability was not possible, authentication instead could be accomplished by the characterization of a wide range of products from the overall area of production of the Moscato wine.

\subsubsection{Milk}

A recent study (Aceto et al. 2017) involved milk, characterized by a quite long production chain, since traceability should be guaranteed from the soil on which the animal feed is cultivated to the final product. Proving the traceability in these conditions is quite challenging due to the animal metabolism that could alter the REEs profile. Two different producers were taken into account, using both artificial feed and hay from their own fields: in both cases the REEs profile proved to be unaltered between soil and hay from the same fields of the producers, while the profile of artificial feed was different. Comparing the profile to that of the corresponding milk, a deep alteration of the REEs was recorded, for both types of feed: an important effect of the animal metabolism was hypothesized in this case, since the digestion in this particular case involves 4 stomachs and an important contribution from water could also be present. The REE profile of fresh milk was compared to that of the same milk undergone the production steps within the local "Centrale del Latte" proving that the elemental profile is not altered by the production chain. In this context, traceability is certainly hampered but authentication can be performed. Samples from "Centrale del Latte" were then compared to samples from other regions: PCA was able to clearly separate the samples in the two groups.

The above reported examples show the importance of «chemical labeling » of food products to support and integrate PDO, GPI etc. labels. In this context Lanthanides appear to work particularly well as traceability fingerprint of the product. This procedure could be coupled to standard documentary traceability to provide a chemical and objective fingerprint of the product.

From the examples provided, it appears clear that traceability must be verified from field to table in each case study: when traceability is not possible since the elemental profile is altered through the production chain, usually authentication can be accomplished. In the case of authentication, usually different chemical fingerprinting tools are used: in this case, data Fusion methods can be applied to merge together different sources of information when dealing with a complex chemical characterization and provide a multivariate model of authentication taking into account all the possible sources of information.

\section{Assays based on REEs}

In spite of the complexity of the overall picture REEs have in living systems, along the years a number of applications in the biomedical field have been designed. Often they appear as real breakthroughs based on the peculiar properties of REEs, something hardly accessible by means of transition and main groups metals.

\section{Luminescent Lanthanide Binding Tags}

The peculiar electronic structures of Lanthanide(III) ions yield intense luminescent signals. Often, to further improve the luminescent signals, small organic fluorophores that absorb in the UV region and transfer the absorbed light to the lanthanide ion are used. The lanthanide complexes display narrow band emission spectra, large Stokes shifts, with lifetimes of the excited states that last from micro- to milliseconds. This allows carrying out time-resolved spectroscopy with the elimination of the background signals that do not last more than $10 \pm 5$ ns. (Rajapakse et al. 2009).

The idea was to develop Lanthanide Binding Tags (LBT) consisting of encoded amino acids easily incorporated into recombinant proteins (Bunzli 2004). They form stable complexes with $\operatorname{Ln}(\mathrm{III})$ ions with physical properties that are useful in biochemical and biophysical investigations. The small size of LBT tags ensure a minimal impact on the structures and functions of proteins to which they are fused. These systems display unique photophysical properties that make them very useful for applications in cell biology and biochemistry, offering information about the topology of multi-enzyme complexes, protein-protein interactions, and protein localization. Most often LBTs have been used as fluorescent probes for in vitro studies and protein purification, exploiting in particular the luminescent properties of terbium(III) and europium(III). 
An interesting application has been recently reported by A. Boffi and coworkers who fused a lanthanide binding tag (LBT) to the C-terminal end of mouse H-chain ferritin, HFt (Calisti et al. 2018). Ferritin displays 24 symmetrically related recognition epitopes for the CD71 receptor (the receptor of Transferrin) instead of the two of classical antibodies or two of transferrin. Overall, the multiplicity of recognition epitopes in ferritin results in a high affinity towards the target receptor (a property often referred to as "multivalence effect") that, in the case of CD71, rivals with typical antibodies affinities. By confocal microscopy and FACS analysis it was shown that HFt-LBT Tb(III) was actively uptaken by selected tumour cell lines.

\section{REE and scintillators for medical imaging}

Scintillators are materials able to efficiently convert high energy radiation or particles into light that can be detected by an electronic sensor such as a photomultiplier tube, photodiode, or silicon photodetector. Scintillating materials find extensive use in the fields of security, defense, high energy physics, and medical diagnostics and imaging. The global market size for inorganic scintillators was estimated to be USD 337.8 million in the year 2016.

In the medical field, these materials are crucial in important and widely spread imaging techniques such as Computed Tomography (CT) and Positron Emission Tomography (PET). In these two cases, the employed scintillators convert
$\mathrm{X}$-rays (CT) or $\gamma$-rays (PET) radiation into visible or, more rarely, UV light that can be easily detected with relatively simple devices. As an example, the scheme of the acquisition process of a positron emission tomograph is shown in Fig. 5.

REE ions (with a few exceptions) are very efficient emitters of light upon excitation from different sources and are very intensively used in the development of luminescent materials. For this reason, they are extremely useful in the field of scintillators. Materials containing lanthanide ions as activators $(\approx 1 \mathrm{~mol} \%)$ give rise to strong light emission in the spectral region from UV to near IR (Fig. 6) upon excitation with ionizing radiation that is by itself very difficult to detect. $\mathrm{Ln}^{3+}$ activated scintillators can therefore efficiently convert X-rays or $\gamma$-rays into light that can be easily and cheaply detected by standard photodetectors (Fig. 6). Moreover, since the ability of absorbing energy from the ionizing radiation (stopping power) depends strongly on the density and the average atomic number of the material, in most cases the scintillator host also contains in its formula relatively heavy lanthanide elements, or Y (that is comprised in the REE group). These host components are not optically active and do not significantly perturb the emission spectrum of the activator ion.

The physical mechanisms underlying the scintillation properties and the CT and PET imaging techniques are described in ref. (Ronda et al. 2016). In the present short review, it is only useful to remark that $\mathrm{CT}$ is able to provide anatomic images of the body, containing 3D information about density differences. On the other hand, PET is a
Fig. 5 Scheme of the acquisition process of a positron emission tomograph (PET) commonly used in cancer diagnostics. Part of the master thesis of Jens Maus (University of Applied Sciences, Dresden, Gemany, 2003), released into the public domain and available on Wikimedia Commons

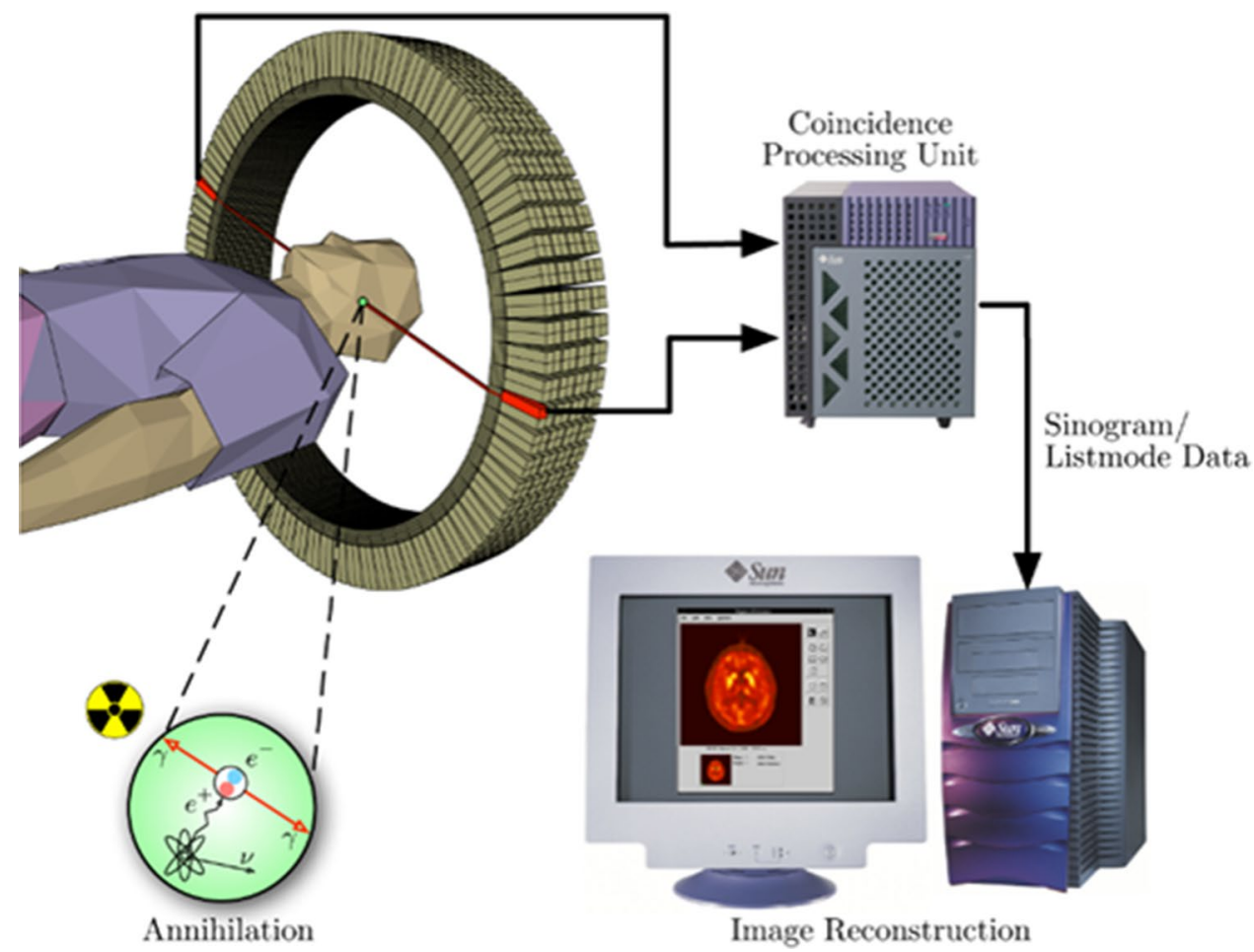




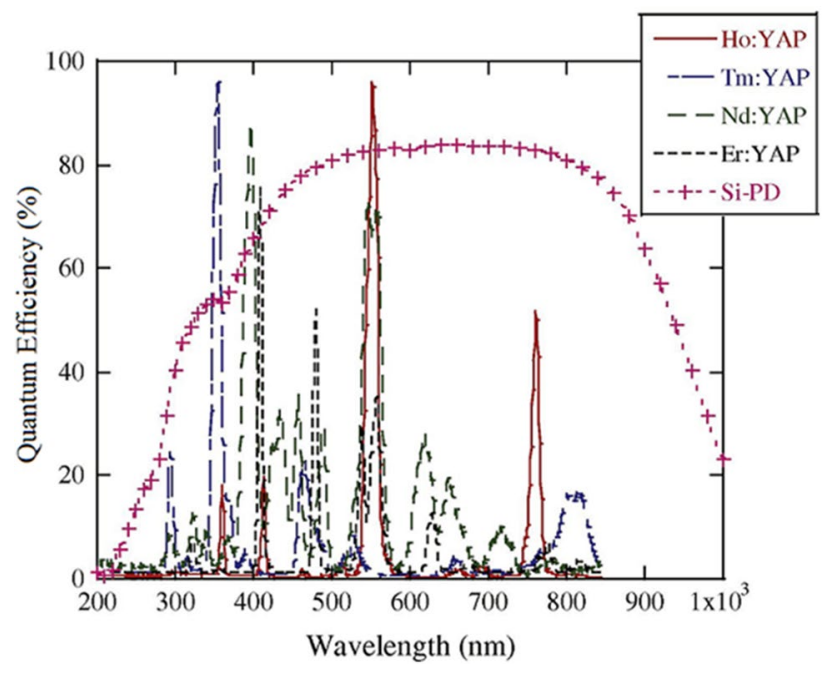

Fig. 6 X-ray induced luminescence spectra of $\mathrm{YAlO}_{3}$ (YAP) scintillating materials activated by the lanthanide ions $\mathrm{Ho}^{3+}, \mathrm{Tm}^{3+}, \mathrm{Nd}^{3+}$ and $\mathrm{Er}^{3+}$, superimposed to the quantum efficiency profile of silicon photodetector (Si-PD) Taken with permission and adapted from $\mathrm{T}$. Yanagida, Opt. Mater. 35 (2013) 1987

functional technique that is based on the injection of a sugar marked with the radioactive isotope ${ }^{18} \mathrm{~F}$. The sugar locates preferentially in areas where increased metabolic activity is present (e.g. cancer cells). The isotope ${ }^{18} \mathrm{~F}$ decays through the emission of a positron, which then collides with an electron giving rise to two collinear $\gamma$-ray photons at an angle of $180^{\circ}$, having an energy of $511 \mathrm{keV}$ (Fig. 5). These photons are then converted to visible or UV light by the scintillator detectors disposed in a circle around the patient and the converted light is detected. A suitable software can then provide functional $3 \mathrm{D}$ images that allow to locate processes taking place in the body.

In the case of $\mathrm{CT}$, some representative scintillators efficiently converting X-rays into visible light are $\mathrm{YAlO}_{3}: \mathrm{Ce}^{3+}$, $(\mathrm{Y}, \mathrm{Gd})_{2} \mathrm{O}_{3}: \mathrm{Eu}^{3+}, \mathrm{Gd}_{2} \mathrm{O}_{2} \mathrm{~S}: \mathrm{Pr}^{3+}$ (the luminescent activator ion is indicated after the colon sign). As for PET, some of the scintillators currently used are $\mathrm{Gd}_{2} \mathrm{SiO}_{5}: \mathrm{Ce}^{3+}$, $\mathrm{Gd}_{3} \mathrm{Al}_{2} \mathrm{Ga}_{3} \mathrm{O}_{12}: \mathrm{Ce}^{3+}, \mathrm{Lu}_{2} \mathrm{SiO}_{5}: \mathrm{Ce}^{3+}$, $(\mathrm{Lu}, \mathrm{Y})_{2} \mathrm{SiO}_{5}: \mathrm{Ce}^{3+}$, $\mathrm{Lu}_{3} \mathrm{Al}_{5} \mathrm{O}_{12}: \mathrm{Ce}^{3+}, \mathrm{Lu}_{3} \mathrm{Al}_{5} \mathrm{O}_{12}: \mathrm{Pr}^{3+}$. It is important to note that PET normally requires the use of relatively large single crystals that constitute a significant amount of the cost of a whole PET equipment, which is extremely expensive.

In conclusion, scintillators based on lanthanides (and in general REE) are extremely important in the field of medical imaging and diagnostics, such as CT and PET. They are strategic materials whose availability and price depend on the REE market, which is strongly dominated by China. Recent developments like Time of Flight Positron Emission Tomography (TOF-PET) require lanthanidebased very fast scintillators. However, TOF-PET provides an outstanding improvement in image quality, allowing to identify small cancer formations, and to detect the disease when other imaging techniques show normal results. Very active research continues in the scientific community in the quest for high-performance scintillator materials based on REE (Qin et al. 2017) that minimize the exposition of the patients to high energy radiation.

\section{Lanthanides and NMR spectra}

The magnetic properties of paramagnetic $\mathrm{Ln}$ (III) ions have been extensively exploited to extract structural information by NMR. The extremely fast electron relaxation of paramagnetic Ln(III) ions (except Gd(III)) (Bertini et al. 1993) makes them suitable as structural probes because they provide minimal nuclear transverse relaxation (that affect line broadening) and longitudinal relaxation enhancements (Bertini et al. 2001a). Paramagnetic relaxation enhancement (PRE) depends on the inverse sixth power of the metal-nucleus distance and therefore is a source of structural information. At the same time, and by the same physical origin that makes electron relaxation extremely efficient, Ln(III) ions are characterized by a sizeable anisotropy of their magnetic susceptibility tensor (Bertini et al. 2001b).

In turn, magnetic susceptibility anisotropy generates significant pseudocontact shifts (PCS) as well

as residual dipolar couplings (RDC) that are observable in NMR experiments (Parigi et al. 2019).

Both PCS and RDC contain structural information (Banci et al. 1996; Banci et al. 1998).

Lanthanide ions can be introduced in proteins in different ways. Among these, there is extensive literature describing metal-substitution in calcium-binding proteins but also the use of lanthanide binding tags chemically attached to proteins have been largely used (Nitsche and Otting 2018).

By preparing multiple samples in each of which a different ion of the lanthanide series is introduced, it is possible to obtain multiple independent PCS datasets that can be used synergistically to generate protein structure ensembles (typically called bundles).

For typical NMR-based determination of protein structure, it is necessary to perform an energetic refinement of such initial bundles to obtain final structures whose geometric quality is suitable for deposition in the PDB.

This task can be tackled by using restrained molecular dynamics simulations (rMD) in explicit solvent. The Florence team has done enormous contributions in this field by developing proper protocols for rMD using multiple PCS datasets as part of the restraints.

They extended the PCS module of the AMBER MD package to handle multiple datasets and tuned a previously developed protocol for NMR structure refinement to 
achieve consistent convergence with PCS restraints (Sala et al. 2016).

\section{Lanthanides and Magnetic Resonance Imaging}

Magnetic Resonance Imaging (MRI) is one of the most important and prominent techniques in diagnostic clinical medicine and biomedical research. Amongst the available imaging modalities, MRI ha the greatest spatial resolution and clinical potential, and can consistently image structures in the millimetre range without the use of ionizing radiation such as that used in X-ray and CT scanning. Traditionally, the imaging procedures have been combined with the use of dedicated contrast media, to improve the visualization of morphology and physiology. This combination of imaging hardware and contrast media was important for the development of modern clinical radiology. MRI is not different in this respect, and the contrast media used are based largely on complexes of $f$-elements (Aime et al. 1998).

A MR image is a topological representation of the distribution of water signal intensities. The contrast is therefore the result of a complex interplay between endogenous factors (proton density, relaxation time, water exchange across biological compartments, etc.) and instrumental parameters essentially liked to the acquisition sequences. On this basis, it was then rather straightforward, in the eighties of the previous century, to identify in the class of the paramagnetic substances the materials to be considered for the design of MRI contrast agents. Nowadays the most commonly used contrast agents are represented by thermodynamic and kinetically stable low molecular weight gadolinium(III) complexes (Fig. 7). The unique magnetic properties of Gd(III) ion (i.e. high magnetic moment associated with the presence of 7 unpaired $f$ electrons and a long electronic relaxation time) together with good chemical characteristics of its complexes with polyaminocarboxylate ligands (i.e. high thermodynamic and kinetic stability, good aqueous solubility, etc.) are instrumental in enhancing the relaxation rate of water protons in tissues (Aime et al. 2005). Gd -based contrast agents are used in about $40 \%$ of all MRI exams and in about $60 \%$ of neuro MRI exams. This leads to about 40 million administrations of Gd-based contrast agents worldwide, corresponding to ca. 50 tons of gadolinium) Wahsner et al. 2019). The used agents are indispensable tools to detect small tumour lesions and to report on abnormalities in excretion pathways. New challenges now are to design systems endowed with improved performance, responsiveness and specific targeting abilities.

Along with the continued dominance of gadolinium, in the last decade or so, there has been increasing interest in the utilization of other lanthanide ions in the new class of MRI contrast agents called para-CEST agents (CEST $=$ Chemical Exchange Saturation Transfer). Unlike Gd-based agents that act on the relaxation rate of water protons, CEST systems affect the intensity of the water resonance upon the transfer of saturated magnetization (Fig. 8). Several moieties can act as source of exchanging protons, namely metal-coordinated water molecules, amide or hydroxyl protons on the ligand and so on, whereas the most considered lanthanide ions are represented by $\mathrm{Eu}(\mathrm{III}), \mathrm{Dy}(\mathrm{III})$ and $\mathrm{Yb}(\mathrm{III})$.
Fig. 7 Some of the most common Gd(III) complexes, clinically used as MRI CAs

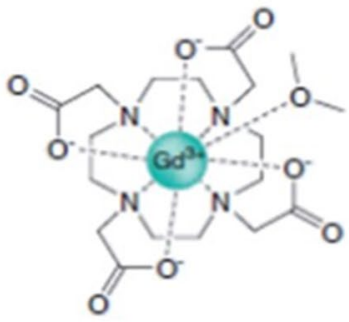

[GdDOTA $\left.\left(\mathrm{H}_{2} \mathrm{O}\right)\right]$

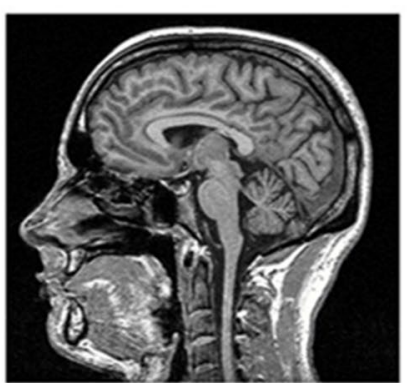

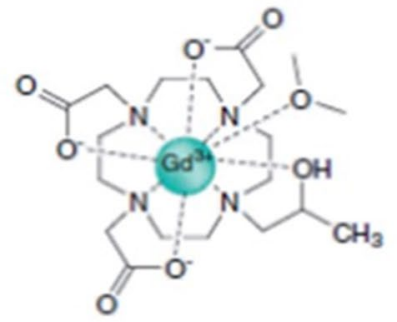

$\left[\mathrm{Gd}(\mathrm{HP}-\mathrm{DO} 3 \mathrm{~A})\left(\mathrm{H}_{2} \mathrm{O}\right)\right]$

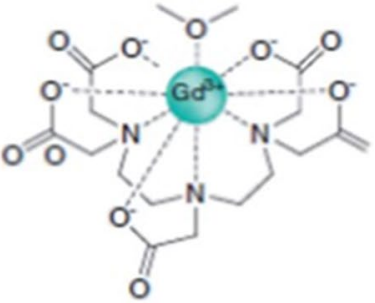

$\left[\operatorname{GdDTPA}\left(\mathrm{H}_{2} \mathrm{O}\right)\right]^{2-}$ 


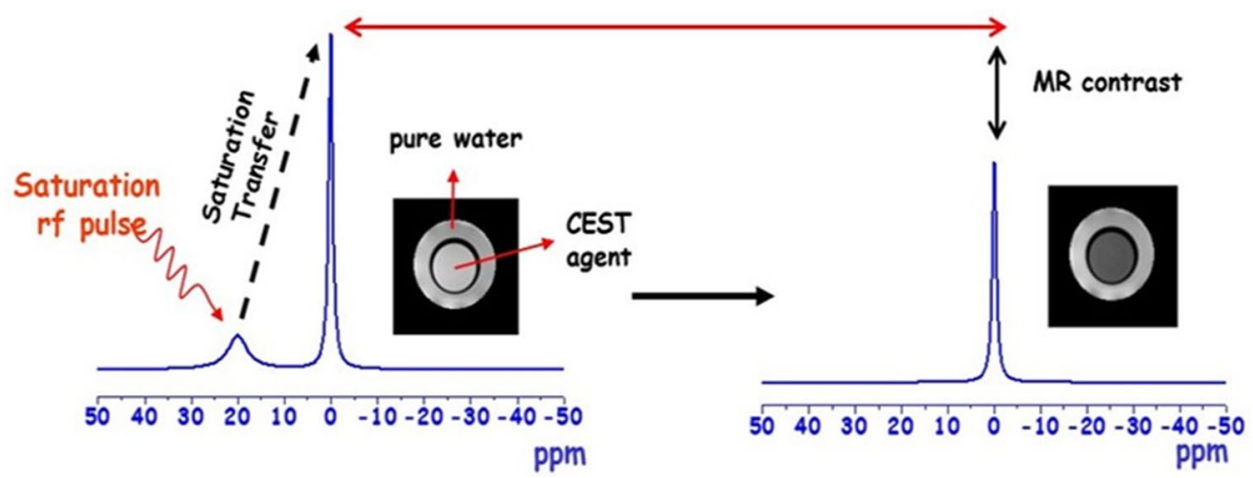

Fig. 8 CEST experiment. Consider a two-pool system where one pool consists of solute protons on the CEST agent and the second consists of water protons with continuous exchange beteen the pools. When a saturation pulse is applied at the frequency of labile protons on the CEST agent (left), saturated CEST protons transfer their loss of polarization to the bulk water because of chemical exchange (right)
These paramagnetic lanthanide ions feature an asymmetric distribution of their electrons in the seven $f$-orbitals that yields large shifts of the proton resonances in their proximity. It follows that a large separation exists between the chemical shifts of the exchangeable pool of protons and the water resonance that allows exploiting large exchange rates before the coalescence of the two signals. Larger exchange rates means increased transfer of saturated protons to the water resonance. Para-CEST agents are therefore frequency encoding agents that result particularly effective as responsive systems (e.g. towards $\mathrm{pH}$, temperature, specific enzymatic activity, etc.) (Terreno et al. 2010).

\section{Lanthanides in mass cytometry}

Technologies assessing the properties of single cells rapidly gain importance in biomedical research. Mass cytometry (Bandura et al. 2009), combining the principles of conventional fluorescence-based flow cytometry and time-of-flight mass spectrometry, permits the characterization of millions of single cells with today more than 50, prospectively more than 100 specific antibodies or other probes (Cossarizza 2019). The lack of autofluorescence artefacts, the negligible signal spillover, and the high dimensionality of data, make mass cytometry an ideal platform for biomarker discovery, cell signalling studies, immune cell profiling in health and disease, and the characterization of complex cellular systems and heterogeneous cell types (Baumgart et al. 2017; Nair et al. 2015; Böttcher et al. 2019; Bendall et al. 2011). The stable labelling of antibodies and other probes with suitable amounts of lanthanides is an essential component of the workflow and an active research field pursued in academia and industry. In practice, a chelate containing a monoisotopic $\mathrm{Ln}$ or a highly enriched $\mathrm{Ln}$ isotopes act as the label for a given antibody (Lou et al. 2007). Besides Ln, mass cytometry also employs reagents based on other elements with a molecular weight of 75-209 Da e.g. palladium and platinum isotopes (Mei et al. 2015; 2016; Schulz and Mei 2019), heavy metal nanoparticles (Schulz et al. 2017; Pichaandi et al. 2019), ruthenium and osmium (Catena et al. 2016; Budzinski et al. 2019), tellurium (Willis et al. 2018), and bismuth (Han et al. 2017). The labelled antibodies are used to stain single-cell suspensions in order to stably bind to their antigens on the outer surface of the cellular membranes, or, after appropriate fixation and permeabilization of the cells, in the cytoplasm and the nucleus. The lanthanides, or commonly selected isotopes of a given lanthanide, are identified and quantitatively determined by analysing the cell suspension by means of ICP-MS (Inductively Coupled Plasma Mass Spectrometry). Thus, through the direct comparison of the amount of metal ions associated to individual cells in the specimen, one can distinguish individual cell types based on antigen expression levels, and determine the composition of cell types in a given sample, each defined by its specific protein expression profile revealed by metal-labelled antibodies. Mass cytometry can be further employed to study the cellular retention of environmental or medical agents containing heavy metal such as the cancer drug cisplatin, or nanoparticles (Guo et al. 2017; Chang et al. 2016; López-Serrano Oliver et al. 2019). Very importantly, the mass cytometry approach has evolved also toward an imaging modality thus allowing merging the information concerning the expression of targeted epitopes with their spatial distribution in the investigated tissue sample (Bodenmiller 2016). Mass cytometry and Imaging mass cytometry are increasingly employed in high-dimensional single-cell studies in translational research, systems biology, and biomarker discovery. 


\section{Concluding remarks}

This survey on the major studies and applications areas concerning REEs in Biology and Medicine strongly suggest that these elements will be increasingly relevant for the understanding of basic biological mechanism as well as in the design of innovative medical solutions. On one hand it will become urgent to get an in-depth understanding of how these elements are already present in living systems and how their mobilization in the environment emerging from their use in high-tech devices and other applications could represent a potential hazard in the forthcoming years. Concern has already been raised for the increase of gadolinium in the water supply in areas of high population density where hospitals make a large use of gadolinium containing contrast agents. Certainly it will be important to find efficient methods to keep under control the distribution of REE in the environment when their increase is dependent from antropic activities. Meanwhile the outstanding achievements from the research involving REEs will bring new astonishing applications in biomedicine that will parallel the outstanding exploitation of these elements in the design of high-tech devices.

Acknowledgements Open access funding provided by Università degli Studi di Torino within the CRUI-CARE Agreement.

Funding Fondazione Donegani is gratefully acknowledged for the support to the workshop.

\section{Compliance with ethical standards}

Conflict of interest The authors declare that they have no conflict of interest.

Ethics approval for research involving human participants and/or animals Not applicable.

Open Access This article is licensed under a Creative Commons Attribution 4.0 International License, which permits use, sharing, adaptation, distribution and reproduction in any medium or format, as long as you give appropriate credit to the original author(s) and the source, provide a link to the Creative Commons licence, and indicate if changes were made. The images or other third party material in this article are included in the article's Creative Commons licence, unless indicated otherwise in a credit line to the material. If material is not included in the article's Creative Commons licence and your intended use is not permitted by statutory regulation or exceeds the permitted use, you will need to obtain permission directly from the copyright holder. To view a copy of this licence, visit http://creativecommons.org/licenses/by/4.0/.

\section{References}

Aceto M (2016) The Use of ICP-MS in Food Traceability. In: Espineira M, Santaclara FJ (eds) Advances in food traceability techniques and technologies: improving quality throughout the food chain. Elsevier, UK, pp 137-164

Aceto M, Baldizzone M, Oddone M (2009) Keeping the track of quality: Authentication and traceability studies on wine. In: O'Byrne P (ed) Red Wine and Health. Nova Science, New York, pp 429-466

Aceto M, Robotti E, Oddone M, Baldizzone M, Bonifacino G, Bezzo G, Di Stefano R, Gosetti F, Mazzucco E, Manfredi M, Marengo E (2013) A traceability study on the Moscato wine chain. Food Chem 138:1914-1922

Aceto M, Musso D, Calà E, Arieri F, Oddone M (2017) Role of lanthanides in the traceability of the milk production chain. J Agric Food Chem 65:4200-4208

Aceto M, Calà E, Musso D, Regalli N, Oddone M (2019) A preliminary study on the authentication and traceability of extra virgin olive oil made from Taggiasca olives by means of trace and ultra-trace elements distribution. Food Chem 298:125047

Agarwal N, Kalra VK (1983) Interaction of lanthanide cations and uranyl ion with the calcium/proton antiport system in Mycobacterium phlei. Biochim Biophys Acta 727:285-292

Aime S, Botta M, Fasano M, Terreno E (1998) Lanthanide(III) chelates for NMR biomedical applications. Chem Soc Rev 27:19-29

Aime S, Botta M, Terreno E (2005) Gd(III)-based contrast agents for MRI. Adv Inorg Chem 57:173-232

Anthony C (2004) The quinoprotein dehydrogenases for methanol and glucose. Arch Biochem Biophys 428:2-9

Ballabio D, Robotti E, Grisoni F, Quasso F, Bobba M, Vercelli S, Gosetti F, Calabrese G, Sangiorgi E, Orlandi M, Marengo E (2018) Chemical profiling and multivariate data fusion methods for the identification of the botanical origin of honey. Food Chem 266:79-89

Banci L, Bertini I, Bren KL, Cremonini MA, Gray HB, Luchinat C, Turano P (1996) The use of pseudocontact shifts to refine solution structures of paramagnetic metalloproteins: Met80Ala cyano-cytochrome $\mathrm{c}$ as an example. J Biol Inorg Chem $1: 117-126$

Banci L, Bertini I, Huber JG et al (1998) Partial orientation of oxidized and reduced cytochrome b5at high magnetic fields: magnetic susceptibility anisotropy contributions and consequences for protein solution structure determination. J Am Chem Soc 120:12903-12909

Bandura DR, Baranov VI, Ornatsky OI, Antonov A, Kinach R, Lou X, Pavlov S, Vorobiev S, Dick JE, Tanner SD (2009) Mass cytometry: technique for real time single cell multitarget immunoassay based on inductively coupled plasma time-of-flight mass spectrometry. Anal Chem 81:6813-6822

Baumgart S, Peddinghaus A, Schulte-Wrede U, Mei HE, Grützkau A (2017) OMIP-034: comprehensive immune phenotyping of human peripheral leukocytes by mass cytometry for monitoring immunomodulatory therapies. Cytometry A. 91:34-38

Bendall SC, Simonds EF, Qiu P et al (2011) Single-cell mass cytometry of differential immune and drug responses across a human hematopoietic continuum. Science 332:687-696

Bertini I, Capozzi F, Luchinat C, Nicastro G, Xia Z (1993) Water proton relaxation for some lanthanide aqua ions in solution. J Phys Chem 97:6351-6354

Bertini I, Janik MBL, Lee YM, Luchinat C, Rosato C (2001a) Magnetic susceptibility tensor anisotropies for a lanthanide ion series in a fixed protein matrix. J Am Chem Soc 123:4181-4188

Bertini I, Luchinat C, Parigi G (2001) Solution NMR of paramagnetic molecules: applications to metallobiomolecules and models. In: Current methods in inorganic chemistry. Elsevier, Amsterdam

Bodenmiller B (2016) Multiplexed epitope-based tissue imaging for discovery and healthcare applications. Cell Syst. 2:225-238

Bogart JA, Lewis AJ, Schelter EJ (2015) DFT study of the active site of the XoxF-type natural, cerium-dependent methanol dehydrogenase enzyme. Chemistry 21:1743-1748 
Böttcher C, Schlickeiser S, Sneeboer MAM et al (2019) Human microglia regional heterogeneity and phenotypes determined by multiplexed single-cell mass cytometry. Nat Neurosci 22:78-90

Budzinski L, Schulz AR, Baumgart S et al (2019) Osmium-labeled microspheres for bead-based assays in mass cytometry. J Immunol 202:3103-3112

Bunzli JC (2004) Luminescent lanthanide probes as diagnostic and therapeutic tools. Met Ions Biol Syst 42:39-75

Calisti L, Trabuco MC, Boffi A, Testi C, Montemiglio LC, des Georges A et al (2018) Engineered ferritin for lanthanide binding. PLoS ONE 13(8):e0201859. https://doi.org/10.1371/journal.pone.02018 59

Catena R, Özcan A, Zivanovic N, Bodenmiller B (2016) Enhanced multiplexing in mass cytometry using osmium and ruthenium tetroxide species. Cytometry A. 89:491-497

Chang Q, Ornatsky OI, Siddiqui I, Straus R, Baranov VI, Hedley DW (2016) Biodistribution of cisplatin revealed by imaging mass cytometry identifies extensive collagen binding in tumor and normal tissues. Sci Rep. 6:36641

Chen Y, Luo Y, Qiu N, Fei H, Sheng L, Wang R, Cao F (2015) $\mathrm{Ce}^{3+}$ induces flavonoids accumulation by regulation of pigments, ions, chlorophyll fluorescence and antioxidant enzymes in suspension cells of Ginkgo biloba L. Plant Cell Tiss Organ Cult 123:283-296

Chistoserdova L (2016) Lanthanides: new life metals? World J Microbiol Biotechnol 32:138

Cossarizza A et al (2019) Guidelines for the use of flow cytometry and cell sorting in immunological studies. Eur J Immunol. 49:1457-1973

Cotruvo JA Jr (2019) The chemistry of lanthanides in biology: recent discoveries, emerging principles, and technological applications. ACS Cent Sci 5:1496-1506

Daumann LJ (2019) Essential and ubiquitous: the emergence of lanthanide metallobiochemistry. Angew Chem Int 58:12795-12802

De Simone G, Polticelli F, Aime S, Ascenzi P (2019) No lanthanidesbased catalysis in eukaryotes. IUBMB Life 71:398-399

Ene CD, Ruta LL, Nicolau I, Popa CV, Iordache V, Neagoe AD, Farcasanu IC (2015) Interaction between lanthanide ions and Saccharomyces cerevisiae cells. J Biol Inorg Chem 20:1097-1107

Fashui H (2002) Study on the mechanism of cerium nitrate effects on germination of aged rice seed. Biol Trace Elem Res 87:191-200

Fitriyanto NA, Fushimi M, Matsunaga M, Pertiwiningrum A, Iwama T, Kawai K (2011) Molecular structure and gene analysis of $\mathrm{Ce}^{3+}$-induced methanol dehydrogenase of Bradyrhizobium sp. MAFF211645. J Biosci Bioeng 111:613-617

Ghosh M, Anthony C, Harlos K, Goodwin MG, Blake C (1995) The refined structure of the quinoprotein methanol dehydrogenase from Methylobacterium extorquens at $1.94 \AA$. Structure 3:177-187

Guo Y, Baumgart S, Stärk HJ, Harms H, Müller S (2017) Mass cytometry for detection of silver at the bacterial single cell level. Front Microbiol 8:1326

Han G, Chen SY, Gonzalez VD, Zunder ER, Fantl WJ, Nolan GP (2017) Atomic mass tag of bismuth-209 for increasing the immunoassay multiplexing capacity of mass cytometry. Cytometry A. 91:1150-1163

Hibi Y, Asai K, Arafuka H, Hamajima M, Iwama T, Kawai K (2011) Molecular structure of $\mathrm{La}^{3+}$-induced methanol dehydrogenaselike protein in Methylobacteriumn radiotolerans. J Biosci Bioeng 111:547-549

Keltjens JT, Pol A, Reimann J, Op den Camp HJ (2014) PQQ-dependent methanol dehydrogenases: rare-earth elements make a difference. Appl Microbiol Biotechnol 98:6163-6183

López-Serrano Oliver A, Haase A, Peddinghaus A, Wittke D, Jakubowski N, Luch A, Grützkau A, Baumgart S (2019) Mass cytometry enabling absolute and fast quantification of silver nanoparticle uptake at the single cell level. Anal Chem 91:11514-11519
Lou X, Zhang G, Herrera I, Kinach R, Ornatsky O, Baranov V, Nitz M, Winnik MA (2007) Polymer-based elemental tags for sensitive bioassays. Angew Chem Int Ed Engl 46:6111-6114

Manfredi M, Robotti E, Quasso F, Mazzucco E, Calabrese G, Marengo E (2018) Fast classification of hazelnut cultivars through portable infrared spectroscopy and chemometrics. Spectrochimica Acta 189:427-435

Marengo E, Aceto M, Robotti E, Liparota MC, Bobba M, Pantò G (2005) Archaeometric characterisation of ancient pottery belonging to the archaeological site of Novalesa Abbey (Piedmont, Italy) by ICPMS and spectroscopic techniques coupled to multivariate statistical tools. Anal Chim Acta 537:359-375

Mei HE, Leipold MD, Schulz AR, Chester C, Maecker HT (2015) Barcoding of live human peripheral blood mononuclear cells for multiplexed mass cytometry. J Immunol. 194:2022-2031

Mei HE, Leipold MD, Maecker HT (2016) Platinum-conjugated antibodies for application in mass cytometry. Cytometry A. 89:292-300

Nair N, Mei HE, Chen SY et al (2015) Mass cytometry as a platform for the discovery of cellular biomarkers to guide effective rheumatic disease therapy. Arthritis Research \& Therapy 17:127

Nakagawa T, Mitsui R, Tani A, Sasa K, Tashiro S, Iwama T, Hayakawa T, Kawai K (2012) A catalytic role of XoxF1 as $\mathrm{La}^{3+}$-dependent methanol dehydrogenase in Methylobacterium extorquens strain AM1. PLoS ONE 7:e50480

Nitsche C and Otting G (2018) Intrinsic and Extrinsic Paramagnetic Probes in Paramagnetism. In Luchinat C, Parigi G, Ravera E (Eds.) Experimental Biomolecular NMR,, RSC, chapter 2

Nojiri M, Hira D, Yamaguchi K, Okajima T, Tanizawa K, Suzuki S (2006) Crystal structures of cytochrome $c_{L}$ and methanol dehydrogenase from Hyphomicrobium denitrificans: structural and mechanistic insights into interactions between the two proteins. Biochemistry 45:3481-3492

Oddone M, Aceto M, Baldizzone M, Musso D, Osella D (2009) Authentication and traceability study of hazelnuts from piedmont, Italy. J Agric Food Chem 57:3404-3408

Parigi G, Ravera E, Luchinat C (2019) Magnetic susceptibility and paramagnetism-based NMR. Prog Nucl Magn Reson Spectrosc 114-115:211-236

Pichaandi J, Zhao G, Bouzekri A et al (2019) Lanthanide nanoparticles for high sensitivity multiparameter single cell analysis. Chem Sci. 10:2965-2974

Picone N, Op den Camp HJ (2019) Role of rare earth elements in methanol oxidation. Curr Opin Chem Biol 49:39-44

Pol A, Barends TR, Dietl A, Khadem AF, Eygensteyn J, Jetten MS, Op den Camp HJ (2014) Rare earth metals are essential for methanotrophic life in volcanic mudpots. Environ Microbiol 16:255-264

Qin X, Xiaowang Liu, Huang W, Bettinelli M, Xiaogang Liu (2017) Lanthanide-activated phosphors based on $4 \mathrm{f}-5 \mathrm{~d}$ optical transitions: theoretical and experimental aspects. Chem Rev 117:4488-4527

Rajapakse HE, Reddy DR, Mohandessi S, Butlin NG, Miller LW (2009) Luminescent terbium protein labels for time-resolved microscopy and screening. Angew Chem Int 48:4990-4992

Ramírez-Olvera SM, Trejo-Téllez LI, García-Morales S, Pérez-Sato JA, Gómez-Merino FC (2018) Cerium enhances germination and shoot growth, and alters mineral nutrient concentration in rice. PLoS ONE 13:e194691

Ronda C, Wieczorek H, Khanin V, Rodnyi P (2016) Review-Scintillators for medical imaging: a tutorial overview. ECS J Solid State Sci Technol 5:R3121-R3125

Sala D, Giachetti A, Luchinat C, Rosato A (2016) A protocol for the refinement of NMR structures using simultaneously pseudocontact shift restraints from multiple lanthanide ions. J Biomol NMR 66:175-185

Schulz AR, Mei HE (2019) Surface barcoding of live PBMC for multiplexed mass cytometry. Methods Mol Biol 1989:93-108 
Schulz AR, Stanislawiak S, Baumgart S, Grützkau A, Mei HE (2017) Silver nanoparticles for the detection of cell surface antigens in mass cytometry. Cytometry A. 91:25-33

Terreno E, Delli Castelli D, Viale A, Aime S (2010) Challenges for molecular magnetic resonance imaging. Chem Rev 110:3019-3042

Wahsner J, Gale EM, Rodríguez-Rodríguez A, Caravan P (2019) Chemistry of MRI contrast agents: current challenges and new frontiers. Chem Rev 119:957-1057

Wang X, Lin Y, Liu D, Xu H, Liu H, Zhao F (2012) Cerium toxicity, uptake and translocation in Arabidopsis thaliana seedlings. J Rare Earth 30:579-585

Williams PA, Coates L, Mohammed F, Gill R, Erskine PT, Coker A, Wood SP, Anthony C, Cooper JB (2005) The atomic resolution structure of methanol dehydrogenase from Methylobacterium extorquens. Acta Crystallogr D Biol Crystallogr 61:75-79

Willis LM, Park H, Watson MWL, Majonis D, Watson JL, Nitz M (2018)

Tellurium-based mass cytometry barcode for live and fixed cells. Cytometry A. 93:685-694

Zhao J, Jin JC, Zhou ZQ, Xia CF, Yang XG, Jiang FL, Dai J, Liu Y (2013) High concentration of gadolinium ion modifying isolated rice mitochondrial biogenesis. Biol Trace Elem Res 156:308-315

Publisher's Note Springer Nature remains neutral with regard to jurisdictional claims in published maps and institutional affiliations. 